\title{
GENERAL SURFACE ENERGY FOR SPINAL CORD AND AORTA SEGMENTATION
}

\author{
Harshit Gupta, Daniel Schmitter, Virginie Uhlmann, and Michael Unser \\ Biomedical Imaging Group \\ Ecole Polytechnique Federale de Lausanne (EPFL), CH-1015 Lausanne, Switzerland
}

\begin{abstract}
We present a new surface energy potential for the segmentation of cylindrical objects in 3D medical imaging using parametric spline active contours (a.k.a. spline-snakes). Our energy formulation is based on an optimal steerable surface detector. Thus, we combine the concept of steerability with spline-snakes that have open topology for semi-automatic segmentation. We show that the proposed energy yields segmentation results that are more robust to noise compared to classical gradient-based surface energies. We finally validate our model by segmenting the aorta on a cohort of 14 real 3D MRI images, and also provide an example of spinal cord segmentation using the same tool.
\end{abstract}

Index Terms - Snakes, segmentation, steerability, splines, aorta, spinal cord

\section{INTRODUCTION}

The development of new 3D techniques for the quantitative evaluation of physiological and medical structures has become essential to establish medical diagnostic of various diseases and abnormalities. Usually, a proper segmentation of the object of interest is crucial to obtain accurate measurements. Typically, the segmentation of complex 3D structures requires a template, which is drawn from an atlas of data that represents a population of patients or healthy subjects [1]. However, when the structures of interest are simpler and exhibit low variability among individuals, prior knowledge can be used to design specialized algorithms. Several elements of interest in the human body, such as vascular structures (e.g., the aorta or coronary arteries) and the spinal cord, have cylindrical topology; a characteristic that can be exploited to construct efficient segmentation algorithms. Segmenting cylindrical structures in medical images is an active field of research. Existing methods mostly rely on meshes, tensors, as well as tracking and path minimization [2-5]. The commonality of these methods is the need for optimizing over a large

H. Gupta, D. Schmitter, V. Uhlmann, and M. Unser are with the Biomedical Imaging Group, École polytechnique fédérale de Lausanne (EPFL), Switzerland. This work was funded by the European Research Council, ERC grant agreement No. 692726 and the Swiss National Science Foundation under Grant and 200020-162343. number of parameters, leading to computationally intensive operations.

In an earlier report [6], we proposed an active contour model [7-10] as a deformable surface to segment structures with cylindrical topology. Our parametric spline surface is defined by a small number of coefficients, which makes it convenient for the design of snake algorithms. To evolve the active surface, we initially used an energy term that was based on the divergence theorem to convert volume integrals into surface integrals, and was therefore restricted to the case of closed surfaces. This approach is not suited for open surfaces as it leads to approximation errors; for instance, in cases where the outgoing and incoming flux through the open parts of our cylinder-like surface do not match.

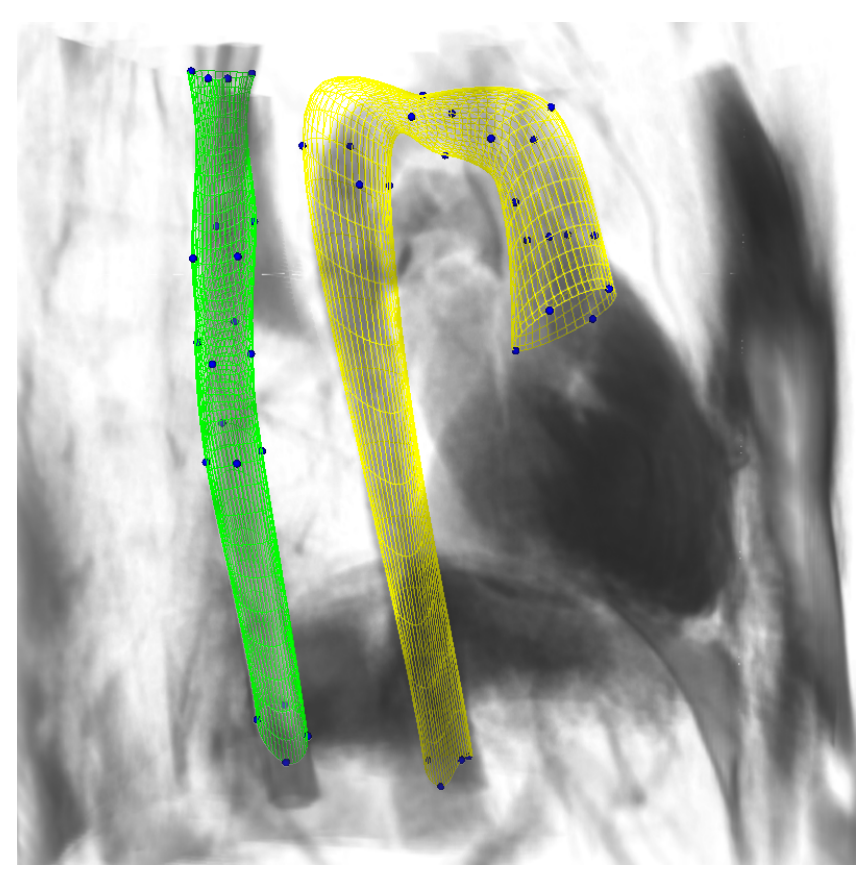

Fig. 1. Segmentation of the spinal cord (left, green) and of the thoracic descending aorta (right, yellow) on real MRI data. The view represents a $3 \mathrm{D}$ rendering of the original image. The blue points on the two colored surfaces are the control points of the cylinder snake and fully specify the surface according to (1). 
Here, we propose a new general surface energy which can be safely used to segment open surfaces, such as the ones with cylindrical topology. It is based on an optimal steerable surface detector $[11,12]$ that identifies the boundary of interest of the object to segment. The surface detector produces an oriented vector field with high-magnitude values located orthogonally to the surface of the object. We construct an energy which, when minimized, aligns the deformable spline surface to the vector field. Using spline surfaces brings the advantage that the energy term only needs to be minimized with respect to a few spline coefficients. Furthermore, the steerable surface detector is more robust to noise than the classical gradient, as observed in synthetic data corrupted by noise. We then demonstrate the efficiency of our pipeline by segmenting the aorta on 14 MRI images of healthy subjects.

\section{CYLINDER SNAKES REVISITED}

In [6], we introduced a deformable parametric surface $\sigma$ : $[0,1] \times[0,1] \rightarrow \mathbb{R}^{3}$ with cylindrical topology given by

$$
\sigma(u, v)=\sum_{k=0}^{M-1} \sum_{l=-1}^{M+1} c[k, l] \varphi_{M, \operatorname{per}}(M u-k) \psi(M v-l),
$$

where $M$ is the number of control points of the surface $\sigma$, $\psi(t)$ is the popular Keys interpolation kernel [13] given by

$$
\psi(t)= \begin{cases}0.5(t+1)(t+2)^{2} & \text { if }-2 \leq t<-1 \\ 0.5\left(2-t^{2}(3 t+5)\right) & \text { if }-1 \leq t<0 \\ 0.5\left((3 t-5) t^{2}+2\right) & \text { if } 0 \leq t<1 \\ -0.5(t-2)^{2}(t-1) & \text { if } 1 \leq t \leq 2\end{cases}
$$

and $\varphi_{M, p e r}(t-k)=\sum_{n=-\infty}^{+\infty} \varphi_{M}(M(t-n)-k)$ is the $M$ periodization of $\varphi_{1}$, which is given by

$$
\varphi_{1}(t)=\lambda_{1} \beta_{\alpha_{1}}(t+2)-\lambda_{2}\left[\beta_{\alpha_{2}}(t+2)+\beta_{\alpha_{2}}(t+1)\right],
$$

with

$$
\begin{aligned}
\lambda_{1}(M) & =\frac{2 \pi^{3}}{M^{2}\left(M \sin \left(\frac{2 \pi}{M}\right)-\pi \cos \left(\frac{2 \pi}{M}\right)-\pi\right)}, \\
\lambda_{2}(M) & =\frac{\pi^{2}\left(2 \pi-M \sin \left(\frac{2 \pi}{M}\right)\right)}{M^{2}\left(\cos \left(\frac{2 \pi}{M}\right)-1\right)\left(M \sin \left(\frac{2 \pi}{M}\right)-\pi \cos \left(\frac{2 \pi}{M}\right)-\pi\right)},
\end{aligned}
$$

where $\hat{\beta}_{\alpha}(\omega)=\Pi_{k=1}^{n} \frac{1-e^{\alpha_{k}-j \omega}}{j \omega-\alpha_{k}}$ is the Fourier transform of the $n^{\text {th }}$ order causal exponential B-spline, whose poles are given by $\alpha_{1}=\left(0,0, \frac{j 2 \pi}{M},-\frac{j 2 \pi}{M}\right)$ and $\alpha_{2}=\left(0, \frac{j 2 \pi}{M},-\frac{j 2 \pi}{M}\right)$. This basis function is interpolatory and can reproduce the functions $\cos (2 \pi t)$ and $\sin (2 \pi t)$ regardless of the number of control points $M$. To generate a perfect cylinder, the control points of the surface $\sigma$ are chosen as

$$
\mathbf{c}[k, l]=\left[\begin{array}{c}
\cos \left[\frac{2 \pi k}{M}\right] \\
\sin \left[\frac{2 \pi k}{M}\right] \\
\frac{l}{M}
\end{array}\right]
$$

Spline-snakes are well-suited for user-interactive segmentation. The snake surface can be easily adjusted by dragging control points with the computer mouse.

\section{OPTIMAL SURFACE DETECTION}

Optimal surface detectors can be tuned to segment objects of interest, when these objects are described by closed surfaces [14]. The detection is based on steerable filters [11, 12]. The filters are designed based on optimality criteria including high signal-to-noise ratio, low localization error of the detected surface element, and suppression of false oscillations. A three-dimensional image $f$ filtered with such a surface detector $h$ produces a vector field $S$. The orientation of $S$ at a fixed location is computed as

$$
\left(\theta^{*}(\mathbf{x}), \phi^{*}(\mathbf{x})\right)=\arg \max _{\theta, \phi}\left(f(\mathbf{x}) * h\left(\mathbf{R}_{\theta, \phi} \mathbf{x}\right)\right)
$$

and its magnitude is obtained by

$$
r^{*}(\mathbf{x})=f(\mathbf{x}) * h\left(\mathbf{R}_{\theta^{*}, \phi^{*}} \mathbf{x}\right)
$$

where $h\left(\mathbf{R}_{\theta, \phi} \mathbf{x}\right)$ is the rotated version of the basis template $h(\mathbf{x})$ rotated by Euler angle $(\theta, \phi)$. A large magnitude of the filter response $r^{*}(\mathbf{x})$ at location $\mathbf{x}$ signals the presence of a surface-like feature, and the corresponding Euler angles $\left(\theta^{*}(\mathbf{x}), \phi^{*}(\mathbf{x})\right)$ give the orientation of the vector that is normal to the surface element at that location. Given an isotropic Gaussian kernel $g$, the surface detector is given by $h(\mathbf{x})=$ $\Delta g(\|\mathbf{x}\|)-5 g_{x x}$, where $g_{x x}$ is the second order partial derivative of $g(\mathbf{x})$ and $\Delta g(\|\mathbf{x}\|)$ is the (rotation-invariant) 3D Laplacian. From [14], we know that a rotation of the filter $h$ by the Euler angles $(\boldsymbol{\theta}, \boldsymbol{\phi})$ results in $h\left(\mathbf{R}_{\theta, \phi} x\right)=\mathbf{v}^{T}\left(\mathbf{A}_{g}(\mathbf{x})\right) \mathbf{v}$, where $\mathbf{v}=(\cos \theta \sin \phi, \cos \theta \cos \phi, \sin \phi)$ is a unit vector specified by the Euler angles, $\mathbf{H}_{g}(\mathbf{x})$ is the 3D Hessian matrix of $g(\mathbf{x})$, and $\mathbf{A}_{g}(\mathbf{x})=\Delta g(\mathbf{x}) \mathbf{I}-\mathbf{5} \mathbf{H}_{g}(\mathbf{x})$. Since the convolution is a linear operation, we have

$$
f(\mathbf{x}) * h\left(\mathbf{R}_{\theta, \phi} \mathbf{x}\right)=\mathbf{v}^{T}\left(\mathbf{A}_{f * g}(\mathbf{x})\right) \mathbf{v} .
$$

The optimal filter response is given by the eigenvector $\mathbf{v}$ of $\mathbf{A}_{f * g}(\mathbf{x})$ that corresponds to the maximum eigenvalue such that $\mathbf{A}_{f * g}(\mathbf{x}) \mathbf{v}^{*}=\lambda_{\max } \mathbf{v}^{*}$. Thus, the optimal orientation and corresponding magnitude of the filter response are given by

$$
\begin{aligned}
r^{*} & =\lambda_{\max }, \\
\left(\theta^{*}, \phi^{*}\right) & =\left(\theta_{v^{*}}, \phi_{v^{*}}\right) .
\end{aligned}
$$

From the properties of steerable filters, any rotated version of $h$ can simply be expressed as a linear combination of a small number of basis filters (9), which significantly relieves the computational overload of the filtering step. 


\section{NEW GENERAL SURFACE ENERGY}

To attract the snake towards the boundaries of the object of interest, we use a general surface-based image energy. Upon minimizing the energy, the snake aligns to the high-magnitude oriented vector field outputted by the optimal steerable surface detector described in Section 3.

\subsection{General Surface Energy}

Given a surface snake $\sigma$, the general surface energy is expressed as

$$
\begin{aligned}
E_{\text {surface }} & =-\iint_{\sigma} S(\sigma) \cdot \mathrm{d} \mathbf{s} \\
& =-\int_{0}^{1} \int_{0}^{1}\left[\left(f(\sigma(u, v)) * h\left(\mathbf{R}_{\theta^{*}, \phi^{*}} \sigma(u, v)\right)\right)\right. \\
& \left.\left(\begin{array}{c}
\cos \theta^{*} \sin \phi^{*} \\
\cos \theta^{*} \cos \phi^{*} \\
\sin \phi^{*}
\end{array}\right) \cdot\left(\mathbf{T}_{1}(u, v) \times \mathbf{T}_{2}(u, v)\right)\right] \mathrm{d} u \mathrm{~d} v,
\end{aligned}
$$

where $\mathbf{T}_{1}(u, v)=\frac{\mathrm{d} \sigma}{\mathrm{d} u}, \mathbf{T}_{2}(u, v)=\frac{\mathrm{d} \sigma}{\mathrm{d} v}$,

$$
\begin{aligned}
& \frac{\partial \sigma}{\partial u}(u, v)=M \sum_{l=0}^{M-1} \sum_{k=-1}^{M+1} \mathbf{c}[k, l] \dot{\varphi}_{M, \text { per }}(M u-k) \psi(M v-l), \\
& \frac{\partial \sigma}{\partial v}(u, v)=M \sum_{l=0}^{M-1} \sum_{k=-1}^{M+1} \mathbf{c}[k, l] \varphi_{M, \text { per }}(M u-k) \dot{\psi}(M v-l) .
\end{aligned}
$$

The construction of the above energy term can be motivated as follows.

1. The surface integral enforces that the surface snake moves towards regions of strong vector field intensity,

2. the dot product between the vector field and the normal to the surface aligns the active surface locally to these regions,

3. the high intensity of the vector field produced by the steerable filter is optimal for the detection of surfaces in the image and therefore yields results superior to the ones obtained with gradient-based techniques.

In essence, the proposed energy aligns the snake to the surface detected by the steerable filter and is reminiscent of the $2 \mathrm{D}$ approach used in [15].

Energy minimization is efficiently achieved by computing its gradient with respect to $\{c[k, l]\}_{k, l \in \mathbb{Z}}$, the control points defining the snake. It is worth mentioning that our energy is independent on the topology and is hence applicable to both open and closed surfaces.
Table 1. Dice indices for the segmentation of the aorta on real MRI data.

\begin{tabular}{c|c}
\hline \hline Image & Result Dice Index \\
\hline \hline 1 & 0.9719 \\
2 & 0.9895 \\
3 & 0.9906 \\
4 & 0.9658 \\
5 & 0.9813 \\
6 & 0.9849 \\
7 & 0.9821 \\
8 & 0.9751 \\
9 & 0.9616 \\
10 & 0.9904 \\
11 & 0.9860 \\
12 & 0.9903 \\
13 & 0.9854 \\
14 & 0.9824 \\
\hline \hline Mean (std) & $0.98(0.02)$ \\
\hline \hline
\end{tabular}

\section{RESULTS AND VALIDATION}

We implemented our general surface energy in combination with the cylinder snake described in Section 2 and illustrate its usefulness in segmenting medical structures that have cylindrical topology.

\subsection{Phantom Data and Robustness to Noise}

First, we test our framework on artificial 3D images of cylinders for which the gold standard is known. Images are corrupted with different levels of additive Gaussian white noise. The cylinder is then segmented using the surface snake optimized either on our general surface energy, or on the more classical gradient-based image energy from [16]. The resulting Dice overlap index are reported in Table 2. As a reminder, the Dice index is defined for two sets $A$ and $B$ as

$$
\text { Dice Index }=\frac{2|A \cap B|}{|A|+|B|} .
$$

The initial position of the snake (before optimization) corresponded to a Dice index of 0.24 .

\subsection{Real Data}

In order to illustrate the efficiency of our method in practice, we applied our framework on 14 3D MRI scans, each originating from a different subject. An example of these data is shown in Figure 1. In each scan, we have segmented the thoracic descending aorta using the general surface energy. Results were then compared to manual segmentations performed by expert clinicians. Results are reported in Table 1. The mean Dice index for the snake initializations is 0.31 . 
Table 2. Comparison of Dice indices when segmenting a cylinder using our generalsurface energy (" proposed") or a classical gradient-based approach (" $\nabla$ ”).

\begin{tabular}{ccc}
\hline \hline SNR [dB] (stdd) & Dice Index (proposed) & Dice Index $(\nabla)$ \\
\hline$\infty(-)$ & 0.97 & 0.81 \\
$9.91(10)$ & 0.96 & 0.73 \\
$0.27(30)$ & 0.96 & 0.84 \\
$-4.17(50)$ & 0.96 & 0.84 \\
$-7.10(70)$ & 0.95 & 0.74 \\
$-9.21(90)$ & 0.94 & 0.83 \\
\hline \hline
\end{tabular}

\section{CONCLUSION}

We introduced a new energy term for surface spline-snakes which is general in the sense that it is applicable to open or closed surfaces. We provide explicit formulas to integrate this energy into an semi-automated active contour segmentation framework relying on cylindrical spline-snakes. We show that our energy term is more robust to noise than the ones that are typically used to detect surfaces and which rely on the classical gradient. To illustrate the usefulness of our approach, we tested and validated it on real data. For the interested reader, we provide demonstration videos at

http://bigwww.epfl.ch/demo/generalizedsurfaceenergy/.

\section{REFERENCES}

[1] Cemil Kirbas and Francis Quek, "A review of vessel extraction techniques and algorithms," ACM Comput. Surv., vol. 36, no. 2, pp. 81-121, June 2004.

[2] B. Li and S.T. Acton, "Active contour external force using vector field convolution for image segmentation," Image Processing, IEEE Transactions on, vol. 16, no. 8, pp. 2096-2106, 2007.

[3] J. Lee, P. Beighley, E. Ritman, and N. Smith, "Automatic segmentation of $3 \mathrm{~d}$ micro-ct coronary vascular images," Medical Image Analysis, vol. 11, no. 6, pp. 630 - 647, 2007.

[4] S. Cetin, A. Demir, A. Yezzi, M. Degertekin, and G. Unal, "Vessel tractography using an intensity based tensor model with branch detection," Medical Imaging, IEEE Transactions on, vol. 32, no. 2, pp. 348-363, Feb 2013.

[5] B. De Leener, S. Kadoury, and J. Cohen-Adad, "Robust, accurate and fast automatic segmentation of the spinal cord," NeuroImage, vol. 98, no. 0, pp. 528 - 536, 2014.

[6] Daniel Schmitter, Christophe Gaudet-Blavignac, Davide Piccini, and Michael Unser, "New parametric 3d snake for medical segmentation of structures with cylindrical topology," in Image Processing (ICIP), 2015 IEEE International Conference on. IEEE, 2015, pp. 276-280.

[7] V. Caselles, R. Kimmel, and G. Sapiro, "Geodesic active contours," International Journal of Computer Vision, vol. 22, no. 1, pp. 61-79, 1997.

[8] F. Precioso and M. Barlaud, "B-spline active contours for fast video segmentation," in Proceedings of the 2001 International Conference on Image Processing, Thessaloniki, Greece, October, 7-10, 2001, pp. 777-780.

[9] T.F. Chan and L.A. Vese, "Active contours without edges," IEEE Transactions on Image Processing, vol. 10, no. 2, pp. 266-277, February 2001.

[10] A. Dufour, R. Thibeaux, E. Labruyere, N. Guillen, and J.-C. Olivo-Marin, "3-D Active meshes: Fast discrete deformable models for cell tracking in 3-D time-lapse microscopy," IEEE Transactions on Image Processing, vol. 20, no. 7, pp. 1925-1937, July 2011.

[11] William T. Freeman and Edward H. Adelson, "The design and use of steerable filters," IEEE Transactions on Pattern Analysis and Machine Intelligence, vol. 13, pp. 891-906, 1991.

[12] M. Jacob and M. Unser, "Design of steerable filters for feature detection using Canny-like criteria," IEEE Transactions on Pattern Analysis and Machine Intelligence, vol. 26, no. 8, pp. 1007-1019, August 2004.

[13] R. Keys, "Cubic convolution interpolation for digital image processing," Acoustics, Speech and Signal Processing, IEEE Transactions on, vol. 29, no. 6, pp. 11531160, Dec 1981.

[14] D. Schmitter, R. Delgado-Gonzalo, G. Krueger, and M. Unser, "Atlas-free brain segmentation in 3D protondensity-like MRI images," in Proceedings of the Eleventh IEEE International Symposium on Biomedical Imaging: From Nano to Macro (ISBI'14), Beijing, People's Republic of China, April 29-May 2, 2014, pp. 629632.

[15] Virginie Uhlmann, Julien Fageot, and Michael Unser, "Hermite snakes with control of tangents," IEEE Transactions on Image Processing, vol. 25, no. 6, pp. 28032816, 2016.

[16] R. Delgado-Gonzalo, N. Chenouard, and M. Unser, "Spline-based deforming ellipsoids for interactive $3 \mathrm{~d}$ bioimage segmentation," IEEE Transactions on Image Processing, vol. 22, no. 10, pp. 3926-3940, October 2013. 